\title{
Aplicação experimental de um modelo de conduta de inspeção sanitária no comércio varejista de alimentos
}

\author{
Experimental application of a sanitary inspection model in the retail food market
}

\author{
Francisco Rafael Martins SOTO ${ }^{1 *}$, Célia Priscilla de Barros CAZZOLA ${ }^{1}$, Eliana de OLIVEIRA ${ }^{1}$, \\ Edson Hideki SAKAGUTI ${ }^{1}$, Fernanda BERNARDI ${ }^{1}$, Dirceu LÚCIO $^{1}$, Nelson YAMASHITA ${ }^{1}$, \\ Sebastião Rodrigues de CAMARGO $^{1}$, Simone de Carvalho BALIAN ${ }^{2}$
}

\begin{abstract}
Resumo
Este trabalho teve por objetivo aplicar um procedimento para diagnóstico e modelo de inspeção higiênico-sanitária em estabelecimentos varejistas de alimentos, no Município de Ibiúna, Estado de São Paulo, Brasil, e analisar os resultados com vistas a atingir os objetivos da vigilância sanitária. O trabalho foi desenvolvido durante seis meses em 19 estabelecimentos varejistas de alimentos classificados como padarias, restaurantes e açougues, sendo que na primeira inspeção realizou-se o diagnóstico da situação, com a identificação das não conformidades e avaliação das condições higiênico-sanitárias dos estabelecimentos e foi obtida a pontuação média de 64. Após esta etapa iniciaram-se retornos programados aos estabelecimentos pela equipe da vigilância sanitária a fim de verificar a evolução das adequações sanitárias. No diagnóstico e em todos os retornos programados foi aplicado roteiro de inspeção e produção de relatórios detalhados destinados aos proprietários. Todos os manipuladores de alimentos dos estabelecimentos inspecionados foram capacitados em relação a conceitos básicos de higiene em alimentos. Os resultados mostraram que, após três retornos de inspeções em cada estabelecimento, 100\% apresentaram-se como satisfatórios, com pontuação média de 90 . O modelo de inspeção sanitária aplicado mostrou-se viável para a promoção da segurança alimentar.
\end{abstract}

Palavras-chave: inspeção sanitária; comércio varejista; alimentos.

\begin{abstract}
The aim of this research was to apply a diagnostic procedure and a model of sanitary inspection in food establishments in the city of Ibiúna, State of São Paulo, Brazil. Results were analyzed in order to achieve sanitary surveillance goals. The work was developed during six months in 19 retail food establishments such as bakeries, restaurants, and butcheries. Firstly, the establishment situation was diagnosed; irregularities were verified according to hygienic-sanitary conditions. An average 64 points was obtained. The next step included new and frequent sanitary surveillance team visits to those establishments in order to verify their sanitary quality adequacies. Inspection guides were used and detailed reports were prepared for the establishment owners. Those who handled food were trained in basic food hygiene. Results indicate that after 3 inspection visits to each establishment, $100 \%$ of them presented satisfactory results with 90 point average. This sanitary inspection model is considered useful to promote food safety.
\end{abstract}

Key words: sanitary inspection; food, retail market.

\section{Introdução}

As doenças de origem alimentar têm sido reconhecidas como um problema de saúde pública de grande abrangência no mundo, causando diminuição da produtividade, perdas econômicas e afetando a confiança do consumidor nos estabelecimentos comerciais de alimentos envolvidos no episódio (RISTOW et al., 2007). Anualmente há milhares de registros pelos órgãos oficiais de saúde pública de intoxicações alimentares e mortalidade de pessoas que ocorrem devido à falha de procedimentos em estabelecimentos do comércio varejista de alimentos (MENNUCCI; SOUZA; CHAABAN, 2007).

A contaminação dos alimentos por microorganismos não pode ser evitada por completo, mas com boas práticas pode ser reduzida, em toda a cadeia produtiva. Durante a manipulação pode haver contaminação por condições precárias de higiene de manipuladores, equipamentos, utensílios, ambiente; por más condições das matérias-primas e ingredientes, ou mesmo más práticas de armazenamento dos produtos acabados (ZANDONADI et al., 2007).

Os manipuladores de alimentos são protagonistas no processo da produção e oferta de alimentos às pessoas, e sua capacitação em todas as etapas é de suma importância para assegurar as condições adequadas dos alimentos que são oferecidos à população (MARQUES et al., 2007).

Os estabelecimentos de preparo e comercialização de alimentos, constituídos por padarias, açougues e restaurantes assumem papel importante na qualidade da alimentação oferecida à população urbana e é competência do serviço de vigilância sanitária (Visa) de

Recebido para publicação em 1/10/2007

Aceito para publicação em 16/1/2008 (002899)

'Secretaria Municipal da Saúde, Centro de Vigilância Sanitária e Controle de Zoonoses "Tereza Rodrigues de Camargo", CP 34, CEP 18150-000, Ibiúna - SP,

E-mail: chicosoto34@gmail.com

${ }^{2}$ Faculdade de Medicina Veterinária e Zootecnia, Universidade de São Paulo, São Paulo - SP

${ }^{*}$ A quem a correspondência deve ser enviada 
alimentos dos municípios supervisionar o funcionamento desses estabelecimentos, no sentido de salvaguardar a saúde pública (RIBEIRO; VAZ; SOUZA, 2007). As ações se concentram, dentre outras, em supervisionar e inspecionar as condições higiênicosanitárias dos estabelecimentos; as práticas de manipulação dos alimentos; a origem das matérias-primas e ingredientes; e as condições dos produtos prontos para consumo em termos de higiene e conservação, fundamentalmente. Porém, são escassas as publicações que oferecem propostas de sistematização no exercício dessas atividades (POTTER; TAUXE, 1997).

Baseado nesta realidade, o presente estudo teve por objetivo implantar experimentalmente um modelo de diagnóstico e de inspeção sanitária no comércio varejista de alimentos do Município de Ibiúna, SP.

\section{Material e métodos}

O trabalho foi desenvolvido entre abril e setembro de 2007 sendo utilizada a metodologia descrita por Soto ${ }^{\text {a }}$ et al., 2006. As inspeções foram programadas em 19 estabelecimentos varejistas de alimentos classificados como açougues (05), padarias (06) e restaurantes (08) localizados na cidade de Ibiúna, SP e representando 100\% destes estabelecimentos na área central urbana do município. Em cada estabelecimento foram efetuadas quatro inspeções, sendo a primeira, definida como o diagnóstico da situação higiênico-sanitária do estabelecimento, e as demais como retornos programados para verificar a evolução do trabalho da Visa. Foi utilizada como base técnica de inspeção a Portaria Estadual CVS no 6 de 10 de março de 1999 (SÃO PAULO (ESTADO) PORTARIA Nº 6 DO CENTRO DE VIGILÂNCIA SANITÁRIA, 1999) e foram anotadas e fotografadas as não conformidades observadas pela equipe técnica da Visa. Esta classificação foi baseada conforme padronização feita pelo Programa de Inspeção em Estabelecimentos na Área de Alimentos (SECRETARIA DE ESTADO DA SAÚDE - SP. CENTRO DE VIGILÂNCIA SANITÁRIA, 1998). Para a obtenção da pontuação, o roteiro de inspeção aplicado foi dividido em cinco blocos. Neste roteiro foi utilizada a Ficha de Inspeção de Estabelecimentos da Área de Alimentos (FIEAA) como instrumento de coleta de dados (SÃO PAULO (ESTADO) RESOLUÇÃO N. 196, 1999). A FIEAA é dividida em quatro partes: a primeira (parte A) refere-se à identificação do estabelecimento, como endereço e sua categoria dentro da área de alimentos. A segunda (parte B) refere-se ao item avaliação e está dividida em cinco blocos, nos quais são mensuradas as conformidades ou não conformidades para: a situação e condição da edificação; equipamentos e utensílios; pessoal na área de produção/manipulação/venda; matérias-primas/produtos expostos à venda; fluxo de produção/ manipulação/venda e controle de qualidade. A terceira (parte C) refere-se à pontuação do estabelecimento. A quarta (parte D) é destinada a possíveis observações pela equipe técnica executora, conclusão final das condições do estabelecimento e agendamento da próxima inspeção.

Em cada bloco da FIEAA (parte C), aplicou-se a Equação 1:

$\frac{T S \times P}{K-N A}$

em que: TS é o somatório dos itens satisfatórios; P, peso do bloco; $\mathrm{K}$, uma constante, utilizada com a finalidade de não penalizar o estabelecimento quando determinado item não for aplicável; e NA, item que não se aplica nas inspeções. Os estabelecimentos foram classificados em: insatisfatórios, quando apresentaram um total de pontos menor do que 50; satisfatórios com restrições, entre 50 e 69 pontos e satisfatórios, quando acima de 70 pontos.

Em todas as inspeções sanitárias, estas foram realizadas por dois agentes de inspeção, um médico veterinário e um nutricionista.

As anotações foram transformadas em relatórios de inspeção sanitária registrados no sistema de informações em Visa (SIVISA, 2007).

Fez-se o levantamento de um total de 80 não conformidades sanitárias baseadas na FIEAA, classificadas como críticas, maiores e menores, sumarizadas na Tabela 1. Estas foram centradas principalmente na parte estrutural (críticas), fluxograma e manipulação de alimentos (maiores), equipamentos e utensílios (menores) e pessoal na área de produção de matérias-primas (maiores).

Todas as informações colhidas foram armazenadas e compiladas em planilhas do Excel, 2002. Quando da presença de não conformidade, ocorreu a definição de prazos para as correções em comum acordo entre a equipe da Visa e o responsável pelo estabelecimento, e estas foram sendo verificadas nas demais inspeções. No caso de não conformidades consideradas maiores (Tabela 1), que poderiam representar risco epidemiológico, o prazo determinado foi exíguo. Nestas situações, a equipe técnica da Visa também realizou a lavratura de auto de infração (Tabela 1) e de imposição de penalidade como: multa ou apreensão de produtos com prazo de validade expirado.

Em cada inspeção, elaborou-se um relatório, sendo uma cópia para o proprietário do estabelecimento, com a avaliação baseada na Portaria Estadual CVS 6/1999. O relatório foi entregue ao estabelecimento, esclarecendo quais eram as não conformidades sanitárias identificadas e por que deveriam ser corrigidas. O prazo acordado entre a equipe da Visa e o proprietário e/ou gerente do estabelecimento foi em média de 28 dias para sanar estas não conformidades. Simultaneamente com o início das inspeções sanitárias, foram realizados dois treinamentos teórico-práticos para os manipuladores de alimentos de todos os estabelecimentos, cujos conteúdos versaram sobre higiene e microbiologia e boas práticas de manipulação e produção de alimentos, desenvolvidos pela equipe técnica da Visa com carga horária de quatro horas para cada curso.

\section{Resultados e discussão}

A Tabela 2 mostra a pontuação média obtida, referente ao diagnóstico efetuado pela equipe técnica da Visa nos estabelecimentos de alimentos, primeira inspeção, (açougues, padarias e restaurantes) sua classificação em porcentagem em insatisfatórios, satisfatórios com restrição e satisfatórios e sua ascensão nas três inspeções subsequentes.

As pontuações relativas à segunda e terceira inspeção mostraram elevação na pontuação média (72 e 77) e redução numérica e em porcentagem ( 4 a 21 e 1 a 5\%) nos pontos relativos às condições insatisfatórias após o primeiro e segundo retorno das inspeções 
Tabela 1. Principais não conformidades avaliadas nos estabelecimentos em relação às condições higiênico-sanitárias, sua classificação em crítica, maior e menor, e a respectiva ação aplicada pela equipe técnica da vigilância sanitária.

\begin{tabular}{|c|c|c|}
\hline Não conformidade & Classificação & Ação \\
\hline Localização adequada, área livre de focos de insalubridade & Crítica & Notificação e orientação \\
\hline Material liso, resistente, impermeável, de fácil limpeza e em bom estado & Menor & Notificação e orientação \\
\hline Existência de proteção contra insetos e roedores & Maior & Auto de infração \\
\hline Ventilação adequada, garantindo o conforto térmico e ambiente livre de fungos & Menor & Notificação e orientação \\
\hline Instalações sanitárias e vestiários adequados & Maior & Auto de infração \\
\hline Lavatórios na área de manipulação adequados & Maior & Auto de infração \\
\hline Destino adequado dos resíduos & Maior & Auto de infração \\
\hline Equipamentos, maquinários e utensílios adequados & Crítica & Notificação e orientação \\
\hline Limpeza e desinfecção adequadas & Maior & Auto de infração \\
\hline Asseio pessoal, lavagem cuidadosa das mãos e estado de saúde controlado & Maior & Auto de infração \\
\hline Procedência, conservação e apresentação adequadas dos alimentos & Maior & Auto de infração \\
\hline
\end{tabular}

sanitárias. Na quarta inspeção está demonstrada a ascensão para a condição satisfatória de todos os estabelecimentos com pontuação média de 90 pontos. Comparando-se os resultados obtidos após a primeira e a quarta inspeção, houve um aumento médio de 26 pontos e $100 \%$ dos estabelecimentos em condição satisfatória.

Apesar do total de estabelecimentos (19) do diagnóstico apresentarem pontuação média de 64 pontos, os resultados mostraram que 50\% das padarias e restaurantes apresentavamse em condições insatisfatórias com pontuação média abaixo de 50 pontos (Tabela 2), necessitando que a equipe da Visa intensificasse as ações nesses estabelecimentos. Acredita-se que o modelo de procedimentos para a prática da vigilância sanitária, no seu aspecto essencialmente construtivo e de educação para se obter e preservar a saúde pública, como já proposto por Soto et al. (2006a), Soto et al. (2006b) mostrouse viável, sendo capaz de alcançar mudanças positivas. Esse modelo foi aplicado e se fundamentou em alguns procedimentos previamente padronizados: 1) inspeção baseada em roteiros para verificação de conformidades/não conformidades, que geram pontuações, classificando o estado geral higiênico do estabelecimento; 2) rigor no cumprimento de prazos para os retornos, mantendo a continuidade do trabalho rigorosamente; 3) argumentação técnica e econômica, esclarecendo "o porquê" de se realizar tal prática de determinada maneira; 4) capacitação técnica dos manipuladores. $\mathrm{O}$ diferencial foi o maior número de estabelecimentos inspecionados e de menor complexidade quando comparado com o trabalho efetuado em supermercados no Município de Ibiúna-SP desenvolvido por Soto et al. (2006a). Houve a necessidade de quatro inspeções em cada estabelecimento, sendo a primeira o diagnóstico e as demais, retornos para que todos atingissem a condição de satisfatórios (Tabela 2). Observouse que os retornos programados criaram nos responsáveis pelos estabelecimentos um compromisso assumido efetivamente entre a Visa e o seu estabelecimento e este fato foi bastante benéfico para que em médio prazo, cada estabelecimento corrigisse falhas e más práticas. Também a programação permitiu à Visa organização, otimização do trabalho, mais foco e esforço dirigido, alcançando mais auto estima e motivação para a equipe. Sistemas de informação como o SIVISA (2007), o gerenciamento contínuo e o uso de softwares agilizando os cálculos foram bastante úteis e válidos em todo o trabalho da equipe.

Este trabalho vem propor uma postura educativa e construtivista às atividades da Visa, sem nunca e, em momento algum, abrir mão do caráter punitivo perante situações de não conformidade que colocam em risco a saúde pública de forma grave. Acredita-se que este procedimento de conduta de trabalho técnico da Visa, desde que continuado, educativo e insistente permite a conquista de boas práticas, por parte dos estabelecimentos de alimentos e também a redução de ocorrências que exigem aplicação das penalidades legais mais drásticas.

Com relação às práticas de capacitação técnica dos manipuladores, permitiu a integração entre setor regulado e regulador, facilitando o diálogo, a compreensão da linguagem técnica e, consequentemente, facilitou, em relação à postura que se mantinha anteriormente ao estudo, a implementação de boas práticas de manipulação de alimentos.

O estudo permitiu também, no decorrer dos trabalhos, observar elevação na auto-estima dos manipuladores, que perceberam o seu trabalho valorizado, reconhecido como importante dentro do estabelecimento. As mudanças comportamentais, de interrelação pessoal, de comprometimento, autoestima e motivação são fundamentais para a implementação de mudanças dentro de uma instituição. Tal assertiva tem sido descrita por vários autores para a promoção da segurança alimentar em estabelecimentos varejistas de alimentos, visto que esta intervenção estará influenciando diretamente nas boas práticas de manipulação e produção de alimentos (CORRÊA,2006a; CORRÊA, 2006b; RODRIGUES et al., 2003; SANTOS, 2006).

Também foi reconhecido pelos proprietários dos estabelecimentos que a importância do trabalho em parceria com a Visa trouxe benefícios diretos no seu produto comercializado, pois mesmo 
Tabela 2. Número $\left(\mathrm{N}^{\circ}\right)$, pontuações médias (PM) e porcentagens (\%) de insatisfatórios (IN), satisfatórios com restrição (SR) e satisfatórios (SA), por categoria de estabelecimento, da primeira (diagnóstico) à quarta inspeção, em relação à condição higiênico-sanitária.

\begin{tabular}{|c|c|c|c|c|c|c|c|c|c|c|c|c|c|c|c|c|c|}
\hline \multirow[t]{2}{*}{ Categoria } & \multicolumn{5}{|c|}{ Diagnóstico (1º Inspeção) } & \multicolumn{4}{|c|}{$2^{\circ}$ Inspeção } & \multicolumn{4}{|c|}{$3^{\circ}$ Inspeção } & \multicolumn{4}{|c|}{$4^{\circ}$ Inspeção } \\
\hline & $\mathrm{N}^{\circ}$ & PM & IN & SR & SA & PM & IN & SR & SA & $\mathrm{PM}$ & IN & SR & SA & PM & IN & SR & SA \\
\hline Açougue & 05 & 82 & $\begin{array}{c}00 \\
00 \%\end{array}$ & $\begin{array}{c}00 \\
00 \%\end{array}$ & $\begin{array}{c}05 \\
100 \%\end{array}$ & 84 & $\begin{array}{c}00 \\
00 \%\end{array}$ & $\begin{array}{c}00 \\
00 \%\end{array}$ & $\begin{array}{c}05 \\
100 \%\end{array}$ & 84 & $\begin{array}{c}00 \\
00 \%\end{array}$ & $\begin{array}{c}00 \\
00 \%\end{array}$ & $\begin{array}{c}05 \\
100 \%\end{array}$ & 90 & $\begin{array}{c}00 \\
00 \%\end{array}$ & $\begin{array}{c}00 \\
00 \%\end{array}$ & $\begin{array}{c}05 \\
100 \%\end{array}$ \\
\hline Restaurante & 08 & 59 & $\begin{array}{c}04 \\
50 \%\end{array}$ & $\begin{array}{c}02 \\
25 \%\end{array}$ & $\begin{array}{c}02 \\
25 \%\end{array}$ & 64 & $\begin{array}{c}03 \\
38 \%\end{array}$ & $\begin{array}{c}03 \\
38 \%\end{array}$ & $\begin{array}{c}02 \\
24 \%\end{array}$ & 79 & $\begin{array}{c}00 \\
00 \%\end{array}$ & $\begin{array}{c}00 \\
00 \%\end{array}$ & $\begin{array}{c}08 \\
100 \%\end{array}$ & 90 & $\begin{array}{c}00 \\
00 \%\end{array}$ & $\begin{array}{c}00 \\
00 \%\end{array}$ & $\begin{array}{c}08 \\
100 \%\end{array}$ \\
\hline
\end{tabular}

tendo que fazer investimentos para corrigir falhas técnicas e operacionais, os resultados alcançados foram de ampla magnitude econômica e de satisfação do cliente, conclusão relatada também por Soto et al. 2006b em um supermercado do Município de Ibiúna.

Acredita-se ainda que a "melhoria" observada neste trabalho pode ser alcançada em outros municípios, utilizando protocolos de inspeção similares. Porém vale considerar que as benfeitorias serão efetivas e mantidas desde que haja continuidade das ações, isto é, se o trabalho da Visa for mantido, com inspeções periódicas e programadas, respaldado por atualizações técnicas dos profissionais, discussões e aprimoramento dos recursos de infraestrutura.

\section{Conclusões}

O protocolo de inspeção sanitária de alimentos proposto neste trabalho mostrou-se um recurso viável, sistematizado, objetivo e útil para a prática da supervisão das boas práticas de fabricação de alimentos pelas Visas dos municípios, desde que a ação punitiva seja sempre mantida como instrumento de salvaguarda da saúde pública em caso de não conformidades classificadas como maiores.

\section{Referências bibliográficas}

CORRÊA, A. A orientação como instrumento de ação em vigilância sanitária direcionada aos manipuladores de alimentos do comércio informal da região sudoeste de Campinas. Revista Brasileira de Vigilância Sanitária, v. 2, n. 1, p. 1-5, 2006a.

CORRÊA, A. C. et al. A vigilância sanitária como orientadora e educadora no controle das doenças transmissíveis por alimentos. Revista Brasileira de Vigilância Sanitária, v. 2, n. 1, p. 6-10, 2006 b.

MARQUES, R. S. et al. Importância do controle da higiene pessoal dos manipuladores de alimentos da merenda escolar do Município de Vitória da Conquista-BA. Revista Higiene Alimentar, v. 21, n. 150, p. 382, 2007.

MENNUCCI, T. A.; SOUZA, T. A. M.; CHAABAN, H. M. A. Prevenção de doenças transmitidas por alimentos em cozinhas residenciais: uma abordagem educativa da vigilância sanitária de Diadema. Revista Higiene Alimentar, v. 21, n. 150, p. 372, 2007.

SÃO PAULO (Estado). Centro De Vigilância Sanitária. Portaria ${ }^{\circ} 6$. Dispõe sobre a aprovação do Regulamento Técnico que Estabelece os Parâmetros e Critérios para o Controle Higiênico-Sanitário em Estabelecimentos de Alimentos. Diário Oficial do Estado de São Paulo, São Paulo, SP, 1999.

POTTER M. E.; TAUXE, R. V. Epidemiology of foodborne diseases: tools and applications. World Health Stater Quality, v. 50, p. 24-29, 1997.

SÃO PAULO (Estado). Resolução n. 196. Dispõe sobre a padronização dos roteiros e guias de inspeção no âmbito do Estado de São Paulo. Diário Oficial do Estado de São Paulo, São Paulo, SP, 1999.

RISTOW, A. M. et al. Avaliação higiênico-sanitária das unidades de alimentação e nutrição localizadas nos Campi de uma Universidade do Rio de Janeiro. Revista Higiene Alimentar, v. 21, n. 150, p. 356, 2007.

RODRIGUES, K. L. et al. Condições higiênico-sanitárias no comércio ambulante de alimentos em Pelotas-RS. Ciência e Tecnologia de Alimentos, v. 23, n. 3, p. 447-452, 2003.

SANTOS, U. R. C. A importância de prática educativa em vigilância sanitária no Estado do Pará. Revista Brasileira de Vigilância Sanitária, v. 2, n. 1, p. 11-16, 2006.

SÃO PAULO (Estado). Secretaria de Estado da Saúde. Centro de Vigilância Sanitária. Programa de inspeção em estabelecimentos da área de alimentos: aspectos operacionais das atividades de inspeção. Versão 03. São Paulo, 1998.

SÃO PAULO (Estado). Secretaria de Estado da Saúde. Centro de Vigilância Sanitária. Sistema de informação em vigilância sanitária. Versão 3.0.8 de 2007.

SOTO, F. R. M. et al. Proposta e análise crítica de um protocolo de inspeção e de condições sanitárias em supermercados do Município de Ibiúna-SP. Revista Brasileira de Epidemiologia, v. 9, n. 2, p. 235-241, 2006a.

SOTO, F. R. M. et al. Resultados da ação da vigilância sanitária de alimentos em um supermercado do Estado de São Paulo. Revista Higiene Alimentar, v. 20, n. 146, p. 21-25, 2006 b.

ZANDONADI, R. P. et al. Atitudes de risco do consumidor em restaurantes de auto-serviço. Revista de Nutrição, v. 20, n. 1, p. 19-26, 2007. 